\title{
Cognitive reappraisal and expressive suppression strategies role in the emotion regulation: an overview on their modulatory effects and neural correlates
}

\author{
Debora Cutuli ${ }^{1,2 *}$ \\ 'Department of Psychology, University "Sapienza" of Rome, Rome, Italy \\ ${ }^{2}$ Laboratory of Experimental and Behavioral Neurophysiology, Santa Lucia Foundation, Rome, Italy
}

Edited by:

Daniela Laricchiuta, Istituto di

Ricovero e Cura a Carattere

Scientifico Santa Lucia Foundation, Italy

Reviewed by:

Giuseppe Curcio, Università degli Studi dell'Aquila, Italy

Maria Beatrice Toro, SCINT Scuola di Specializzazione in Psicoterapia

Cognitivo Interpersonale, Italy

${ }^{*}$ Correspondence:

Debora Cutuli, Department of

Psychology, University "Sapienza"

of Rome, Via dei Marsi 78, 00185

Rome, Italy

e-mail: debora.cutuli@uniroma1.it
Individuals regulate their emotions in a wide variety of ways. In the present review it has been addressed the issue of whether some forms of emotion regulation are healthier than others by focusing on two commonly used emotion regulation strategies: cognitive reappraisal (changing the way one thinks about potentially emotion-eliciting events) and expressive suppression (changing the way one behaviorally responds to emotion-eliciting events). In the first section, experimental findings showing that cognitive reappraisal has a healthier profile of short-term affective, cognitive, and social consequences than expressive suppression are briefly reported. In the second section, individual-difference findings are reviewed showing that using cognitive reappraisal to regulate emotions is associated with healthier patterns of affect, social functioning, and well-being than is using expressive suppression. Finally, brain structural basis and functional activation linked to the habitual usage of cognitive reappraisal and expressive suppression are discussed in detail.

Keywords: emotion regulation, cognitive reappraisal, expressive suppression, brain volume, brain activation

\section{INTRODUCTION}

The number of studies on emotion regulation has dramatically increased in the past two decades. These studies strengthened our knowledge on how the effectiveness of emotion regulation is crucial for different aspects of healthy affective and social adaptation (Gross, 2001; John and Gross, 2004). Further, dysregulation of emotions typically characterizes mood and anxiety disorders (Gross and Thompson, 2007).

Two major emotion regulation strategies that have been particularly studied are cognitive reappraisal and expressive suppression (Gross and John, 1998). In particular, cognitive reappraisal is defined as the attempt to reinterpret an emotion-eliciting situation in a way that alters its meaning and changes its emotional impact (Lazarus and Alfert, 1964; Gross and John, 2003). Expressive suppression is defined as the attempt to hide, inhibit or reduce ongoing emotion-expressive behavior (Gross and Levenson, 1993; Gross and John, 2003).

Based on an analysis of how emotions unfold over time, it has been argued that cognitive reappraisal and expressive suppression have their primary impact at different points of the emotiongenerative process (Figure 1; Gross, 2001; Gross and John, 2003). Specifically, cognitive reappraisal is an antecedent-focused strategy that acts before the complete activation of emotion response tendencies has taken place. It thus might be expected to modify the entire temporal course of the emotional response before emotion responses have been completely generated. Expressive suppression is a response-focused strategy that intervenes once an emotion is already under way and after the behavioral responses have already been fully generated. It thus might be expected to require repeated efforts to manage emotional responses as they continually arise, challenging the individual's resources.

The usage of cognitive reappraisal allows to implement and produce interpersonal behavior that is appropriately focused on social interaction and is perceived by the others as emotionally engaging and responsive. At odds, expressive suppression comes relatively late in the emotion-generative process and principally modifies the behavioral aspect of the emotional responses, without reducing the subjective and physiological experience of negative emotion, which is not directly targeted by suppression and may thus continue to linger and accumulate unresolved. As expressive suppression comes late in the emotion-generative process, it requires the individual to effortfully manage emotional responses as they constantly occur. These repeated efforts deplete cognitive resources to the detriment of social performances and create a sense of discrepancy between inner experience and outer expression in the individual (Higgins, 1987). The final effect of this sense of inauthenticity can lead to negative feelings about the self, making more difficult the establishment of emotionally close relationships and rather contributing to avoidant, diverted and anxious relational behaviors (Sheldon et al., 1997; John and Gross, 2004).

In the following sections, experimental findings on cognitive reappraisal and expressive suppression will be briefly analyzed. Then, individual-difference findings on the dispositional usage 


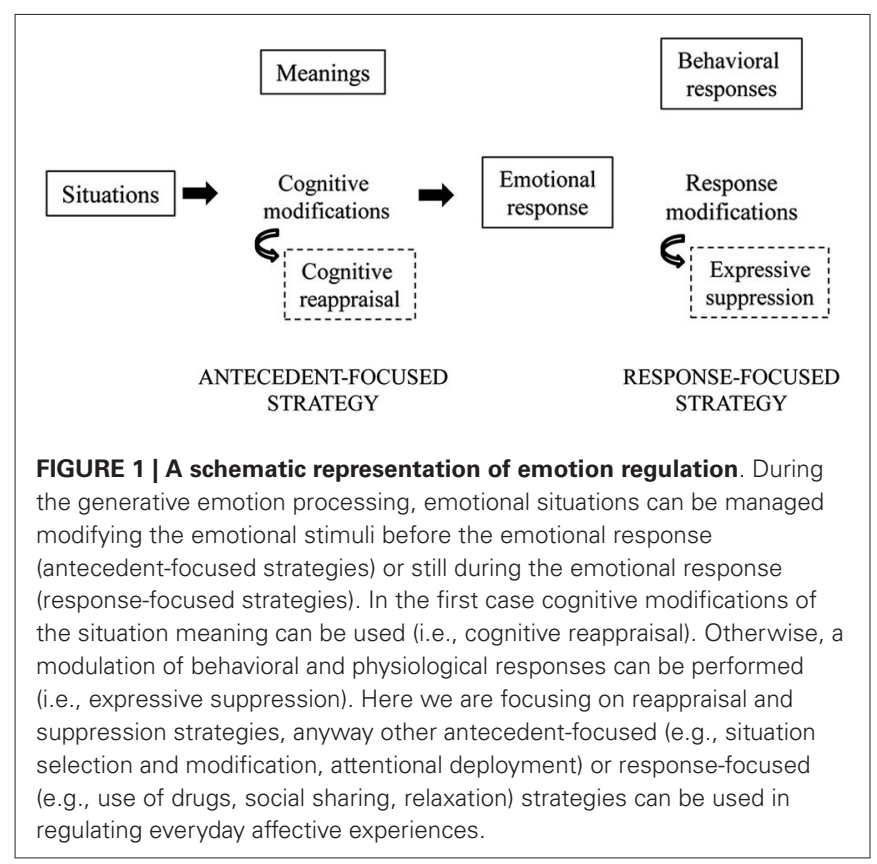

of these two strategies will be taken into account. Finally, brain structural basis and functional activation linked to the habitual usage of cognitive reappraisal and expressive suppression will be discussed in detail.

\section{EXPERIMENTAL STUDIES}

In experimental studies, participants are exposed to emotioneliciting situations and randomly assigned to use cognitive reappraisal or expressive suppression strategies or to act naturally (control condition). Experimental studies use powerful research designs: in fact, by manipulating emotion-regulatory processes directly, they can demonstrate the immediate causal effects of particular strategies on dependent variables of interest, such as affective, cognitive and social consequences.

Overall experimental studies have demonstrated that cognitive reappraisal has a positive impact in the affective domain by decreasing negative emotion experience and negative emotion behavioral expression without any increase in physiological activation. At odds, suppression has a negative impact decreasing positive emotion experience and leaving unaltered the subjective negative emotion experience and exacerbating physiological activation (Gross and Levenson, 1993, 1997; Gross, 2002; Mauss et al., 2005; Hayes et al., 2010; Brans et al., 2013).

Cognitively, reappraisal results in unaltered or enhanced behavioral memory performance, while expressive suppression impairs memory performances (Richards and Gross, 1999, 2000; Dillon et al., 2007; Sheppes and Meiran, 2007, 2008; Hayes et al., 2010). Memory advantage for cognitive reappraisal may be subserved by the levels-of-processing effect (Dillon et al., 2007), which is characterized by deeper cognitive analysis of stimuli (Craik and Lockhart, 1972).

In experimental studies on the effects of emotion regulation strategies in social contexts, one member of each dyad is generally asked either to suppress, reappraise or to interact naturally with their conversation partner. When interacting with a person who was using suppression, subjects experienced more stress (i.e., greater increases in blood pressure) than when interacting with a person using reappraisal (Butler et al., 2003; Richards et al., 2003). Thus, while reappraisal has not detrimental effects, the cognitive costs of expressive suppression may concurr to compromise social functioning, as the suppressor fails to take up information needed to respond appropriately to the others and appears not tuned with the flow of the interaction.

\section{INDIVIDUAL DIFFERENCE STUDIES}

Since experimental studies cannot account for the long-term, cumulative consequences of using particular regulatory strategies for the individual's emotional life, relationships and wellbeing, a complementary, correlational research approach was used. To this aim Gross and John (2003) developed a self-report questionnaire, the Emotion Regulation Questionnaire (ERQ), to assess individual differences in the usage of habitual, dispositional cognitive reappraisal and expressive suppression. Studies using ERQ have shown that the habitual use of these strategies varies systematically between individuals and is stable in time (Gross and John, 2003). Furthermore, cognitive reappraisal and expressive suppression resulted scarcely related to intelligence, social desirability and personality traits, but highly related to the constructs of inauthenticity, coping with stress and mood management (John and Gross, 2004).

Affectively, the use of cognitive reappraisal in everyday life is related to greater experience and expression of positive emotions and lesser experience and expression of negative emotions. By contrast, individuals frequently using expressive suppression experience and express less positive emotions, without differences in the negative ones (Gross and John, 2003; Abler et al., 2010; Larsen et al., 2012). However, expressive suppression may increase negative affect through its strict link with inauthenticity, specifically leading to feel bad about the self and even to depressive symptoms (John and Gross, 2004).

Cognitively, reappraisal has not effects on mnesic performances, while suppression is negatively related to memory, in particular for socially relevant information (Richards and Gross, 2000; Egloff et al., 2006; Hayes et al., 2010; Moore and Zoellner, 2012). In the domains of interpersonal functioning and well-being, cognitive reappraisal was interestingly associated with better psychological health. In fact, individuals who habitually use reappraisal showed lower symptoms of depression, were more satisfied and optimistic, and had higher self-esteem, environmental mastery levels, personal growth, self-acceptance, coping skills, sense of autonomy as well as better interpersonal relationships (Garnefski et al., 2001; John and Gross, 2004). At odds, suppressors feel to have less social support, worse coping abilities, lower life satisfaction, self-esteem, optimistic attitude about the future, higher avoidance and lack of close social relationships and support, all factors increasing the risk for depressive symptoms (Sheldon et al., 1997; John and Gross, 2004). Anyway, interesting recent studies demonstrated that culture has to be a moderator variable of emotion regulation, being the relation between expressive suppression and negative 
indicators of mental health stronger in the Western culture than in the Eastern one (Soto et al., 2011; Hu et al., 2014).

\section{NEURAL CORRELATES OF COGNITIVE REAPPRAISAL AND EXPRESSIVE SUPPRESSION}

As the habitual use of emotion regulation strategies shows stable individual differences, it could be possible that these strategies, either as a consequence (i.e., pre-existing individual volume differences lead to differences in emotion regulation) or precondition (i.e., brain region volumes are affected by the usage of emotion regulation strategies) are associated with individual differences in brain volumes and functional activation. Several studies have investigated the underlying neurobiological substrates of cognitive reappraisal and expressive suppression usage.

Following an overview of the studies on brain structural and functional variations associated to the use of cognitive reappraisal and expressive suppression is presented.

\section{BRAIN STRUCTURAL STUDIES}

In a magnetic resonance imaging (MRI) study, Welborn et al. (2009) investigated the relation between sex differences in orbitofrontal cortex (OFC) subregions and affective individual differences in healthy adults. As previously reported (Gross and John, 2003), women reported using suppression less frequently than did men. Volume differences based on participants' gender were also identified with men showing larger left planum temporal and women showing larger ventromedial prefrontal cortex (vmPFC), right lateral OFC, cerebellum and basal ganglia. Strikingly, vmPFC (but not OFC) volume was positively related to individual differences in cognitive reappraisal and negatively related to expressive suppression usage. Further, vmPFC volume fully mediated sex differences in emotion suppression and partly in cognitive reappraisal.

In another region of interest (ROI)-based neuroimaging study, Giuliani et al. (2011a) found a positive correlation between cognitive reappraisal and the volume of the dorsal anterior cingulate cortex (dACC), but not the ventral ACC, in healthy female subjects. No relations between dACC volume and expressive suppression, negative affect or age were found. Given that expressive suppression is an emotion regulation strategy that requires interoceptive and emotional awareness, the role of anterior insula in this process was further investigated (Giuliani et al., 2011b). It was demonstrated that anterior insula volume positively correlates to expressive suppression, but not with cognitive reappraisal and negative affect. These findings are consistent with the idea that trait patterns of emotion processing are related to brain structure and indicate that individual differences in cognitive reappraisal are related to different dACC volumes, while individual differences in expressive suppression are related to different anterior insula volumes.

Using an exploratory whole brain approach, Kühn et al. (2011) examined the structural correlates of the habitual use of expressive suppression of emotions. They found a positive correlation of right dorsomedial prefrontal cortex (dmPFC) volume with expressive suppression, but no association of any other brain area with cognitive reappraisal. As expected on the basis of the important role that dmPFC plays in self-control and voluntary inhibition of action (Brass and Haggard, 2007; Brody et al., 2007; Campbell-Meiklejohn et al., 2008; Kühn et al., 2009), the response-focused emotion regulation strategy of expressive suppression is associated with increased gray matter volume in the dmPFC. Even if it is not possible to rule out that the increased dmPFC volume in subjects with expressive suppression strategies is an a priori condition rather than a consequence of behavior, it could be speculated that expressive suppression is under internal control as consequence of the internalization of societal norms, customs and manners that govern the adequate or undesirable emotional expressions.

Recently, using a voxel-based morphometry (VBM) in a large sample of young individuals it was analyzed the association of gray matter volumes of the a priori ROIs, including amygdala, insula, dACC/paracingulate cortex, medial and lateral PFC, with cognitive reappraisal and expressive suppression usage as well as neuroticism (Hermann et al., 2013a). Interestingly, a positive association of cognitive reappraisal and neuroticism with amygdala volume was observed. Furthermore, expressive suppression resulted positively associated with $\mathrm{dACC} /$ paracingulate cortex and medial PFC gray matter volume. These findings underline the role of the amygdala in individual differences in cognitive reappraisal usage as well as neuroticism that was not found in previous studies. Additionally, the association of expressive suppression usage with larger volumes of the dACC/paracingulate cortex and medial PFC underpins the role of these regions in regulating emotion-expressive behavior. It is evident that Hermann et al. (2013a) did not replicate previous results regarding greater dACC (Giuliani et al., 2011a) and vmPFC (Welborn et al., 2009) volume in frequent using cognitive reappraisers, and larger insula (Giuliani et al., 2011b) and smaller vmPFC (Welborn et al., 2009) volume in individuals frequently using expressive suppression. In contrast, the positive correlation of expressive suppression with dACC/paracingulate cortex and with vmPFC gray matter volume is in line with the involvement of dmPFC in the network linked to the inhibition of actions (Kühn et al., 2009).

Although somewhat conflicting, overall brain structural studies demonstrate that distinct brain structural variations of gray matter volume in the amygdala, insula, dACC, vmPFC and dmPFC might underlie individual differences in cognitive reappraisal and expressive suppression usage. However, a replication of these results is still missing because most of the abovementioned studies focused on different brain regions. Additionally, methodological factors (e.g., VBM vs. ROI approach) as well as sample characteristics (e.g., gender and age of participants) prevent a reasonable comparison of the results.

\section{BRAIN FUNCTIONAL STUDIES}

The neural basis of emotion regulation processes have been further investigated by several functional neuroimaging studies by manipulating emotion regulation strategies (Ochsner and Gross, 2005). Generally, negative affective pictures are used and participants are trained to reduce the emotional impact of the pictures by using cognitive reappraisal. It is well known that not all individuals experiencing adverse experiences develop anxiety disorders, as result of individual differences in the regulation 
of negative emotions. Anyway, a more frequent use of habitual (dispositional) cognitive reappraisal in daily life has been shown to be more adaptive. Interestingly, the down-regulation of negative emotions through cognitive reappraisal is indicated by increased activation of medial and lateral PFC along with a diminished activation of emotional arousal-related brain structures as amygdala and insula (Ochsner and Gross, 2005; Ochsner et al., 2012).

Furthermore, dispositional reappraisal has been associated with reduced insula, hippocampus and amygdala as well with stronger dACC and dorsolateral PFC activation in response to aversive emotional stimuli (i.e., pictures or faces; Drabant et al., 2009; Carlson and Mujica-Parodi, 2010; Hayes et al., 2010; Vanderhasselt et al., 2013; Hermann et al., 2014).

Recently, the correlation of habitual cognitive reappraisal usage with stronger down-regulation of amygdala activation during instructed emotion regulation was reported also in a group of patients with remitted depression and healthy controls by using functional MRI (fMRI; Kanske et al., 2012). Hermann et al. (2013b) found that dental phobic individuals with higher dispositional cognitive reappraisal scores showed a reduced activation of the right dmPFC and increased activation of the right vmPFC and the lateral OFC over the course of symptom provocation. Cognitive reappraisal was a predictor of habituation during exposure to phobic stimuli rather than symptom severity. Given that extinction learning as well as cognitive reappraisal are crucial components of exposure-based cognitive-behavioral therapy (CBT) of phobias, the findings by Hermann et al. (2013b) point out for the special importance of considering individual differences in general cognitive reappraisal abilities of phobic patients prior to exposure sessions and to improve these abilities if necessary in order to strengthen the (long-term) outcome of CBT.

Up-to-date few studies examined the neural correlates of expressive suppression in response to emotional stimuli (Ohira et al., 2006; Goldin et al., 2008; Hayes et al., 2010; Vanderhasselt et al., 2013). Ohira et al. (2006) demonstrated a reduced amygdala activation during suppression of emotions. In a further PET study, Goldin et al. (2008) demonstrated increased PFC, insula and amygdala activation during the suppression of disgust facial reactions in response to disgust-eliciting film clips. Individual differences in expressive suppression usage have been further associated with higher amygdala activation when inhibiting responses to sad vs. happy facial expressions (Vanderhasselt et al., 2013). Suppressing facial expressions in response to negative picture engaged bilateral insular cortex, supramarginal gyrus and middle frontal gyrus (Hayes et al., 2010).

In parallel with gray matter volume studies, taken together these studies on the functional activation during cognitive reappraisal and expressive suppression confirm that differential activation of the amygdala, insula, dACC, PFC and OFC might underlie individual differences in the use of different emotional strategies.

\section{DISCUSSION}

Altogether experimental and individual difference studies underpin the crucial role of cognitive reappraisal and expressive suppression in adaptive as well as dysfunctional emotional processing and regulation. Furthermore, brain structural and functional studies depict a resulting brain network constituted by target regions for several emotional regulation processes. Namely, the amygdala has a crucial role in emotion regulation as it processes sensory information from the thalamus and somatosensory cortex and has bidirectional projections with hippocampus (emotional memories) and hypothalamus (physiological activation). The regulation of emotional processes is modulated by an rich net of interconnections among amygdala, insula (enteroception, sense of self) and the cortico-subcortical circuits of the OFC (saliency evaluation of emotional state, selection of adequate behaviors) and ACC (emotional state interpretation, motivated behavior). Also PFC (executive functions, cognitive elaboration) indirectly participates in the emotional regulation through its connections with OFC.

Not by chance association between amygdala gray matter volume and anxiety-related traits/states have been reported in numerous studies in healthy subjects (Barrós-Loscertales et al., 2006; Tottenham et al., 2010; van der Plas et al., 2010; Gerritsen et al., 2012) as well as altered activation and volume in the amygdala are common findings in mood and anxiety disorders (Etkin and Wager, 2007; Drevets et al., 2008; Irle et al., 2010; Atmaca, 2011; Kempton et al., 2011; Sacher et al., 2012). Furthermore, reduced activation of the vmPFC along with amygdala hyperactivation and a dysfunctional recruitment of ACC and dmPFC has been observed in patients with specific phobia and post-traumatic stress disorder (Schienle et al., 2007; Hermann et al., 2009; Milad et al., 2009), most likely indicating reduced cognitive control of emotional reactions. Interestingly, phobic individuals more frequently using cognitive reappraisal have an increased vmPFC activation during extinction learning and recall (Hermann et al., 2013b), probably related to a stronger extinction learning as following a successful CBT (Schienle et al., 2007).

The top-down emotional control network via cognitive reappraisal engages also OFC (Ochsner and Gross, 2005; Hermann et al., 2013b). By contrast, habitual bottom-up use of expressive suppression rely more heavily on the anterior insula (Giuliani et al., 2011a) and dACC/paracingulate cortex and medial PFC volume (Hermann et al., 2013a) as well as on increased insula, PFC and amygdala activation (Ohira et al., 2006; Goldin et al., 2008; Hayes et al., 2010; Vanderhasselt et al., 2013). In this neural correlates pattern the role of the insula emerges, not only in primarily supporting interoception and monitoring emotional awareness and outward emotional expression, but also as a relay point between the bottom-up signals from brain regions involved in emotional responding and inward emotional state, like the amygdala, and bottom-up signals from other regions involved in cognitive regulation and regulation goals, like the PFC (Nunn et al., 2008).

\section{CONCLUSIONS}

As conclusive considerations, further studies are required to outline more in depth the relations among structural and functional data, trait and state emotion regulation and their interactions. In fact, given the strict relationship between expressive suppression, depression and stress-related symptoms (Moore et al., 2008), the 
question of whether this strategy is a vulnerability or causal factor remains still open. Otherwise, to evaluate its long term effects on anxiety, depression or other pathologies innovative clinical interventions could be designed training clients to cognitive reappraisal or even positive reappraisal, a recent trying to incorporate meditation mindfulness into cognitive therapy (Garland et al., 2009; Hanley and Garland, 2014).

Finally, another direction for the future studies is to carry out longitudinal researches that, allowing repeated observations of the effects of using particular emotion regulation strategies, would help to understand the causal order of effects of the habitual use of cognitive reappraisal or expressive suppression.

\section{REFERENCES}

Abler, B., Hofer, C., Walter, H., Erk, S., Hoffmann, H., Traue, H. C., et al. (2010). Habitual emotion regulation strategies and depressive symptoms in healthy subjects predict fMRI brain activation patterns related to major depression. Psychiatry Res. 183, 105-113. doi: 10.1016/j.pscychresns.2010.05.010

Atmaca, M. (2011). Review of structural neuroimaging in patients with refractory obsessive-compulsive disorder. Neurosci. Bull. 27, 215-220. doi: 10.1007/s12264011-1001-0

Barrós-Loscertales, A., Meseguer, V., Sanjuán, A., Belloch, V., Parcet, M. A., Torrubia, R., et al. (2006). Behavioral inhibition system activity is associated with increased amygdala and hippocampal gray matter volume: a voxel-based morphometry study. Neuroimage 33, 1011-1015. doi: 10.1016/j.neuroimage. 2006.07.025

Brans, K., Koval, P., Verduyn, P., Lim, Y. L., and Kuppens, P. (2013). The regulation of negative and positive affect in daily life. Emotion 13, 926-939. doi: 10. 1037/a0032400

Brass, M., and Haggard, P. (2007). To do or not to do: the neural signature of selfcontrol. J. Neurosci. 27, 9141-9145. doi: 10.1523/jneurosci.0924-07.2007

Brody, A. L., Mandelkern, M. A., Olmstead, R. E., Jou, J., Tiongson, E., Allen, V., et al. (2007). Neural substrates of resisting craving during cigarette cue exposure. Biol. Psychiatry 62, 642-651. doi: 10.1016/j.biopsych.2006.10.026

Butler, E. A., Egloff, B., Wilhelm, F. H., Smith, N. C., Erickson, E. A., and Gross, J. J. (2003). The social consequences of expressive suppression. Emotion 3, 48-67. doi: 10.1037/1528-3542.3.1.48

Campbell-Meiklejohn, D. K., Woolrich, M. W., Passingham, R. E., and Rogers, R. D. (2008). Knowing when to stop: the brain mechanisms of chasing losses. Biol. Psychiatry 63, 293-300. doi: 10.1016/j.biopsych.2007.05.014

Carlson, J. M., and Mujica-Parodi, L. R. (2010). A disposition to reappraise decreases anterior insula reactivity during anxious anticipation. Biol. Psychol. 85, 383-385. doi: 10.1016/j.biopsycho.2010.08.010

Craik, F. I. M., and Lockhart, R. S. (1972). Levels of processing: a framework for memory research. J. Verbal Learning Verbal Behav. 11, 671-684. doi: 10.1016/ s0022-5371(72)80001-x

Dillon, D. G., Ritchey, M., Johnson, B. D., and LaBar, K. S. (2007). Dissociable effects of conscious emotion regulation strategies on explicit and implicit memory. Emotion 7, 354-365. doi: 10.1037/1528-3542.7.2.354

Drabant, E. M., McRae, K., Manuck, S. B., Hariri, A. R., and Gross, J. J. (2009). Individual differences in typical reappraisal use predict amygdala and prefrontal responses. Biol. Psychiatry 65, 367-373. doi: 10.1016/j.biopsych.2008.09.007

Drevets, W. C., Price, J. L., and Furey, M. L. (2008). Brain structural and functional abnormalities in mood disorders: implications for neurocircuitry models of depression. Brain Struct. Funct. 213, 93-118. doi: 10.1007/s00429-008-0189-x

Egloff, B., Schmukle, S. C., Burns, L. R., and Schwerdtfeger, A. (2006). Spontaneous emotion regulation during evaluated speaking tasks: associations with negative affect, anxiety expression, memory and physiological responding. Emotion 6, 356-366. doi: 10.1037/1528-3542.6.3.356

Etkin, A., and Wager, T. D. (2007). Functional neuroimaging of anxiety: a metaanalysis of emotional processing in PTSD, social anxiety disorder and specific phobia. Am. J. Psychiatry 164, 1476-1488. doi: 10.1176/appi.ajp.2007. 07030504

Garland, E., Gaylord, S., and Park, J. (2009). The role of mindfulness in positive reappraisal. Explore (NY) 5, 37-44. doi: 10.1016/j.explore.2008.10.001
Garnefski, N., Kraaij, V., and Spinhoven, P. (2001). Negative life events, cognitive emotion regulation and emotional problems. Pers. Individ. Dif. 30, 1311-1327. doi: 10.1016/s0191-8869(00)00113-6

Gerritsen, L., Rijpkema, M., van Oostrom, I., Buitelaar, J., Franke, B., Fernández, G., et al. (2012). Amygdala to hippocampal volume ratio is associated with negative memory bias in healthy subjects. Psychol. Med. 42, 335-343. doi: 10. 1017/s003329171100122x

Giuliani, N. R., Drabant, E. M., Bhatnagar, R., and Gross, J. J. (2011a). Emotion regulation and brain plasticity: expressive suppression use predicts anterior insula volume. Neuroimage 58, 10-15. doi: 10.1016/j.neuroimage.2011. 06.028

Giuliani, N. R., Drabant, E. M., and Gross, J. J. (2011b). Anterior cingulate cortex volume and emotion regulation: is bigger better? Biol. Psychol. 86, 379-382. doi: 10.1016/j.biopsycho.2010.11.010

Goldin, P. R., McRae, K., Ramel, W., and Gross, J. J. (2008). The neural bases of emotion regulation: reappraisal and suppression of negative emotion. Biol. Psychiatry 63, 577-586. doi: 10.1016/j.biopsych.2007.05.031

Gross, J. J. (2001). Emotion regulation in adulthood: timing is everything. Curr. Dir. Psychol. Sci. 10, 214-219. doi: 10.1111/1467-8721.00152

Gross, J. J. (2002). Emotion regulation: affective, cognitive and social consequences. Psychophysiology 39, 281-291. doi: 10.1017/s0048577201393198

Gross, J. J., and John, O. P. (1998). Mapping the domain of emotional expressivity: multi-method evidence for a hierarchical model. J. Pers. Soc. Psychol. 74, 170191. doi: 10.1037//0022-3514.74.1.170

Gross, J. J., and John, O. P. (2003). Individual differences in two emotion regulation processes: implications for affect, relationships and well-being. J. Pers. Soc. Psychol. 85, 348-362. doi: 10.1037/0022-3514.85.2.348

Gross, J. J., and Levenson, R. W. (1993). Emotional suppression: physiology, selfreport and expressive behavior. J. Pers. Soc. Psychol. 64, 970-986. doi: 10. 1037//0022-3514.64.6.970

Gross, J. J., and Levenson, R. W. (1997). Hiding feelings: the acute effects of inhibiting positive and negative emotions. J. Abnorm. Psychol. 106, 95-103. doi: 10.1037//0021-843x.106.1.95

Gross, J. J., and Thompson, R. A. (2007). "Emotion regulation: conceptual foundations," in Handbook of Emotion Regulation, ed J. J. Gross (New York: Guilford Press), 3-24.

Hanley, A. W., and Garland, E. L. (2014). Dispositional mindfulness co-varies with self-reported positive reappraisal. Pers. Individ. Dif. 66, 146-152. doi: 10.1016/j. paid.2014.03.014

Hayes, J. P., Morey, R. A., Petty, C. M., Seth, S., Smoski, M. J., McCarthy, G., et al. (2010). Staying cool when things get hot: emotion regulation modulates neural mechanisms of memory encoding. Front. Hum. Neurosci. 4:230. doi: 10. 3389/fnhum.2010.00230

Hermann, A., Bieber, A., Keck, T., Vaitl, D., and Stark, R. (2013a). Brain structural basis of cognitive reappraisal and expressive suppression. Soc. Cogn. Affect. Neurosci. doi: 10.1093/scan/nst130. [Epub ahead of print].

Hermann, A., Keck, T., and Stark, R. (2014). Dispositional cognitive reappraisal modulates the neural correlates of fear acquisition and extinction. Neurobiol. Learn. Mem. 113, 115-124. doi: 10.1016/j.nlm.2014. 03.008

Hermann, A., Leutgeb, V., Scharmüller, W., Vaitl, D., Schienle, A., and Stark, R. (2013b). Individual differences in cognitive reappraisal usage modulate the time course of brain activation during symptom provocation in specific phobia. Biol. Mood Anxiety Disord. 3:16. doi: 10.1186/2045-5380-3-16

Hermann, A., Schäfer, A., Walter, B., Stark, R., Vaitl, D., and Schienle, A. (2009). Emotion regulation in spider phobia: role of the medial prefrontal cortex. Soc. Cogn. Affect. Neurosci. 4, 257-267. doi: 10.1093/scan/nsp013

Higgins, E. T. (1987). Self-discrepancy: a theory relating self and affect. Psychol. Rev. 94, 319-340. doi: 10.1037//0033-295x.94.3.319

Hu, T., Zhang, D., Wang, J., Mistry, R., Ran, G., and Wang, X. (2014). Relation between emotion regulation and mental health: a meta-analysis review. Psychol. Rep. 114, 341-362. doi: 10.2466/03.20.pr0.114k22w4

Irle, E., Ruhleder, M., Lange, C., Seidler-Brandler, U., Salzer, S., Dechent, P., et al. (2010). Reduced amygdalar and hippocampal size in adults with generalized social phobia. J. Psychiatry Neurosci. 35, 126-131. doi: 10.1503/jpn. 090041

John, O. P., and Gross, J. J. (2004). Healthy and unhealthy emotion regulation: personality processes, individual differences and life span development. J. Pers. 72, 1301-1333. doi: 10.1111/j.1467-6494.2004.00298.x 
Kanske, P., Heissler, J., Schönfelder, S., and Wessa, M. (2012). Neural correlates of emotion regulation deficits in remitted depression: the influence of regulation strategy, habitual regulation use and emotional valence. Neuroimage 61, 686693. doi: 10.1016/j.neuroimage.2012.03.089

Kempton, M. J., Salvador, Z., Munafò, M. R., Geddes, J. R., Simmons, A., Frangou, S., et al. (2011). Structural neuroimaging studies in major depressive disorder. Meta-analysis and comparison with bipolar disorder. Arch. Gen. Psychiatry 68, 675-690. doi: 10.1001/archgenpsychiatry.2011.60

Kühn, S., Gallinat, J., and Brass, M. (2011). "Keep calm and carry on”: structural correlates of expressive suppression of emotions. PLoS One 6:e16569. doi: 10. 1371/journal.pone.0016569

Kühn, S., Haggard, P., and Brass, M. (2009). Intentional inhibition: how the "vetoarea" exerts control. Hum. Brain Mapp. 30, 2834-2843. doi: 10.1002/hbm.20711

Larsen, J. K., Vermulst, A. A., Eisinga, R., English, T., Gross, J. J., Hofman, E., et al. (2012). Social coping by masking? Parental support and peer victimization as mediators of the relationship between depressive symptoms and expressive suppression in adolescents. J. Youth Adolesc. 41, 1628-1642. doi: 10.1007/s10964012-9782-7

Lazarus, R. S., and Alfert, E. (1964). Short-circuiting of threat by experimentally altering cognitive appraisal. J. Abnorm. Psychol. 69, 195-205. doi: 10. 1037/h0044635

Mauss, I. B., Levenson, R. W., McCarter, L., Wilhelm, F. H., and Gross, J. J. (2005). The tie that binds? Coherence among emotion experience, behavior and physiology. Emotion 5, 175-190. doi: 10.1037/1528-3542.5.2.175

Milad, M. R., Pitman, R. K., Ellis, C. B., Gold, A. L., Shin, L. M., Lasko, N. B., et al. (2009). Neurobiological basis of failure to recall extinction memory in posttraumatic stress disorder. Biol. Psychiatry 66, 1075-1082. doi: 10.1016/j. biopsych.2009.06.026

Moore, S. A., and Zoellner, L. A. (2012). The effects of expressive and experiential suppression on memory accuracy and memory distortion in women with and without PTSD. J. Exp. Psychopathol. 3, 368-392. doi: 10.5127/jep.024411

Moore, S. A., Zoellner, L. A., and Mollenholt, N. (2008). Are expressive suppression and cognitive reappraisal associated with stress-related symptoms? Behav. Res. Ther. 46, 993-1000. doi: 10.1016/j.brat.2008.05.001

Nunn, K., Frampton, I., Gordon, I., and Lask, B. (2008). The fault is not in her parents but in her insula-a neurobiological hypothesis of anorexia nervosa. Eur. Eat. Disord. Rev. 16, 355-360. doi: 10.1002/erv.890

Ochsner, K. N., and Gross, J. J. (2005). The cognitive control of emotion. Trends Cogn. Sci. 9, 242-249. doi: 10.1016/j.tics.2005.03.010

Ochsner, K. N., Silvers, J. A., and Buhle, J. T. (2012). Functional imaging studies of emotion regulation: a synthetic review and evolving model of the cognitive control of emotion. Ann. N Y Acad. Sci. 1251, E1-E24. doi: 10.1111/j.1749-6632. 2012.06751.x

Ohira, H., Nomura, M., Ichikawa, N., Isowa, T., Iidaka, T., Sato, A., et al. (2006). Association of neural and physiological responses during voluntary emotion suppression. Neuroimage 29, 721-733. doi: 10.1016/j.neuroimage.2005.08.047

Richards, J. M., Butler, E., and Gross, J. J. (2003). Emotion regulation in romantic relationships: the cognitive consequences of concealing feelings. J. Soc. Pers. Relat. 20, 599-620. doi: 10.1177/02654075030205002

Richards, J. M., and Gross, J. J. (1999). Composure at any cost? The cognitive consequences of emotion suppression. Pers. Soc. Psychol. Bull. 25, 1033-1044. doi: $10.1177 / 01461672992511010$

Richards, J. M., and Gross, J. J. (2000). Emotion regulation and memory: the cognitive costs of keeping one's cool. J. Pers. Soc. Psychol. 79, 410-424. doi: 10. 1037/0022-3514.79.3.410
Sacher, J., Neumann, J., Funfstuck, T., Soliman, A., Villringer, A., and Schroeter, M. L. (2012). Mapping the depressed brain: a meta-analysis of structural and functional alterations in major depressive disorder. J. Affect. Disord. 140, 142148. doi: 10.1016/j.jad.2011.08.001

Schienle, A., Schäfer, A., Hermann, A., Rohrmann, S., and Vaitl, D. (2007). Symptom provocation and reduction in patients suffering from spider phobia: an fMRI study on exposure therapy. Eur. Arch. Psychiatry Clin. Neurosci. 257, 486-493. doi: 10.1007/s00406-007-0754-y

Sheldon, K. M., Ryan, R. M., Rawsthorne, L. J., and Ilardi, B. (1997). Trait self and true self: cross-role variation in the big-five personality traits and its relations with psychological authenticity and subjective well-being. J. Pers. Soc. Psychol. 73, 1380-1393. doi: 10.1037//0022-3514.73.6.1380

Sheppes, G., and Meiran, N. (2007). Better late than never? On the dynamics of online regulation of sadness using distraction and cognitive reappraisal. Pers. Soc. Psychol. Bull. 33, 1518-1532. doi: 10.1177/0146167207305537

Sheppes, G., and Meiran, N. (2008). Divergent cognitive costs for online forms of reappraisal and distraction. Emotion 8, 870-874. doi: 10.1037/a001 3711

Soto, J. A., Perez, C. R., Kim, Y. H., Lee, E. A., and Minnick, M. R. (2011). Is expressive suppression always associated with poorer psychological functioning? A cross-cultural comparison between European Americans and Hong Kong Chinese. Emotion 11, 1450-1455. doi: 10.1037/a0023340

Tottenham, N., Hare, T. A., Quinn, B. T., McCarry, T. W., Nurse, M., Gilhooly, T., et al. (2010). Prolonged institutional rearing is associated with atypically large amygdala volume and difficulties in emotion regulation. Dev. Sci. 13, 46-61. doi: 10.1111/j.1467-7687.2009.00852.x

Vanderhasselt, M. A., Baeken, C., Van Schuerbeek, P., Luypaert, R., and De Raedt, R. (2013). Inter-individual differences in the habitual use of cognitive reappraisal and expressive suppression are associated with variations in prefrontal cognitive control for emotional information: an event related fMRI study. Biol. Psychol. 92, 433-439. doi: 10.1016/j.biopsycho.2012.03.005

van der Plas, E. A., Boes, A. D., Wemmie, J. A., Tranel, D., and Nopoulos, P. (2010). Amygdala volume correlates positively with fearfulness in normal healthy girls. Soc. Cogn. Affect. Neurosci. 5, 424-431. doi: 10.1093/scan/nsq009

Welborn, B. L., Papademetris, X., Reis, D. L., Rajeevan, N., Bloise, S. M., and Gray, J. R. (2009). Variation in orbitofrontal cortex volume: relation to sex, emotion regulation and affect. Soc. Cogn. Affect. Neurosci. 4, 328-339. doi: 10. $1093 /$ scan/nsp028

Conflict of Interest Statement: The author declares that the research was conducted in the absence of any commercial or financial relationships that could be construed as a potential conflict of interest.

Received: 15 July 2014; accepted: 01 September 2014; published online: 19 September 2014.

Citation: Cutuli D (2014) Cognitive reappraisal and expressive suppression strategies role in the emotion regulation: an overview on their modulatory effects and neural correlates. Front. Syst. Neurosci. 8:175. doi: 10.3389/fnsys.2014.00175

This article was submitted to the journal Frontiers in Systems Neuroscience.

Copyright (c) 2014 Cutuli. This is an open-access article distributed under the terms of the Creative Commons Attribution License (CC BY). The use, distribution or reproduction in other forums is permitted, provided the original author(s) or licensor are credited and that the original publication in this journal is cited, in accordance with accepted academic practice. No use, distribution or reproduction is permitted which does not comply with these terms. 